\title{
SURGICAL MANAGEMENT OF BURULI ULCER DISEASE: A FOUR- YEAR EXPERIENCE FROM FOUR ENDEMIC DISTRICTS IN GHANA
}

\author{
E.J.K. ADU ${ }^{1}$, E. AMPADU ${ }^{2}$ and D. ACHEAMPONG ${ }^{3}$ \\ ${ }^{1}$ Department of Surgery, School of Medical Sciences, College of Health Sciences, Kwame Nkrumah Univer- \\ sity of Science and Technology, Kumasi, Ghana ${ }^{2}$ National Buruli Ulcer Control Programme, Korle Bu, Ac- \\ cra ${ }^{3}$ Accident and Emergency Unit, Komfo Anokye Teaching Hospital, Kumasi, Ghana
}

Corresponding Author: Dr. Emmanuel J.K. Adu

E-mail address: $\underline{\text { aduemmanuel@hotmail.com }}$

Conflict of Interest: None declared

\section{SUMMARY}

Background: Mycobacterium ulcerans (MU) disease causes extensive destruction of tissues leaving large ulcers on the body. Management which consisted of surgical excision of the lesions is gradually being replaced with chemotherapy.

Objective: To study the impact on surgery of prior treatment of MU disease with rifampicin and streptomycin.

Study Design: Retrospective, from September 2004 to September 2009.

Setting: Asunafo, Amansie West, Ahafo Ano and Amansie Central districts of Ghana.

Methods: Patients who have completed 8 weeks or a minimum of 4 weeks treatment with rifampicin and streptomycin but have unhealed lesions were selected for surgery.

Results: 132 patients had surgery for MU disease; 51 at Tepa (Ahafo Ano); 36 at Agroyesum (Amansie West); 16 from Jacobu (Amansie Central); 29 from Goaso (Asunafo) districts. Their ages ranged from 4 to 98 years, with mean age of 29.90 years, standard deviation of 20.74. Sites involved were: head and neck $1(0.74 \%)$, upper limb 40 (29.63\%), lower limb $92(68.15 \%)$, trunk $2(1.48 \%)(\mathrm{N}=135)$. The clinical forms were: papule $1(0.74 \%)$, nodule $2(1.48 \%)$, oedematous lesion $4(2.96 \%)$, osteomyelitis 2 $(1.48 \%)$, ulcers $124(91.85 \%)$, contractures 2 (1.48\%). 139 surgical procedures were performed: excision $25(18.11)$, skin grafting $36(26.1 \%)$, excision and skin grafting $54(39.1 \%)$, debridem net 10 $(7.2 \%)$, sequestrectomy $2(1.4 \%)$, regrafting 10 $(7.2 \%)$, release of contractures $2(1.4 \%)$.

Conclusion: Treatment of MU disease with rifampicin and streptomycin improved the condition and minimised the extent of surgery. Combination of surgery and antibiotics is necessary to prevent the development contractures.

Keywords: Mycobacterium ulcerans, Buruli ulcer, skin grafting, contracture, antibiotics

\section{INTRODUCTION}

Buruli ulcer is a specific infection caused by Mycobacterium ulcerans ${ }^{1}$ (MU) an acid fast bacillus ${ }^{2}$. Analysis of its complete genome sequence has revealed the presence of a set of three large genes which produces mycolactone $^{3}$, a toxin which has cytotoxic and immunosuppressive properties. ${ }^{2,4}$ Infection with MU often leads to extensive destruction of the skin and soft tissue with the formation of large ulcers, usually on the legs or arms ${ }^{5}$ and other exposed parts of the body such as the head and neck. ${ }^{6}$

MU disease has been classified into non-ulcerative and ulcerative forms. ${ }^{4}$ The non-ulcerative forms include the papule, nodule, plaque, and oedematous forms. The ulcer, when fully developed, has undermined edges and is indurated peripherally. The floor of the ulcer may have white cotton-wool-like slough ${ }^{4}$. The lesion is usually painless unless there is secondary infection. ${ }^{2}$

Until the introduction of antibiotic treatment in 2004 by the World Health Organisation ${ }^{7}$ surgical treatment was the accepted definitive treatment. ${ }^{8}$ Currently it is known that between $30-50 \%$ of cases can be treated solely with antibiotics - namely rifampicin -streptomycin combination. $^{3,9}$ However some cases require a combination of surgery and antibiotics; even in such cases it is recommended that antibiotics treatment should precede surgery by a minimum of four weeks., 9

An additional classification of MU disease was introduced $^{3,7}$ with the institution of antibiotic treatment. The lesions are classified into three categories according to size:

Category I: a single lesion $<5 \mathrm{~cm}$ in diameter

Category II: a single lesion between $5 \mathrm{~cm}$ and $15 \mathrm{~cm}$ in diameter

Category III: a single lesion $>15 \mathrm{~cm}$ in diameter, multiple lesions, lesions at critical sites (e.g. eye, breast, genitalia), and osteomyelitis. 
Category I lesions may completely heal with antibiotics treatment; some category II lesions may completely heal with antibiotics. Category III lesions require both antibiotics and surgery; however multiple small lesions and lesions located at critical sites may heal with antibiotics alone. ${ }^{3}$

Buruli ulcer occurs most frequently in tropical and subtropical areas that are marshy or near lakes or rivers. ${ }^{2,3,10}$ In Africa, Benin, Cote d'Ivoire and Ghana are the most endemic countries. ${ }^{3 .}$ Cases in Ghana were identified in all 10 regions and in 90 (81.8\%) of the then 110 districts in a national case search in 1999. ${ }^{5}$

As a strategic intervention, the national Buruli ulcer programme organises regular community surgical outreach services to some endemic districts; and it was during such outreach programmes to Asunafo, Amansie-West, Ahafo-Ano and Amansie Central districts in Ghana, between 2005 to 2009, that this study was undertaken. The objective of the study was to observe and document the effect of the WHO recommended intervention in the treatment of Buruli ulcer disease using antibiotics and surgery.

\section{PATIENTS AND METHODS}

A four-year retrospective study to determine the impact of prior antibiotic therapy on surgery for Buruli Ulcer disease was undertaken within the period from September 2005 to September 2009. This study was undertaken as part of the normal surgical outreach programmes of the National Buruli Ulcer Control Programme (NBUCP) to Goaso Government Hospital, Asunafo; St. Martin's Catholic Hospital atAgroyesum, Amansie West; Tepa District Hospital, Ahafo Ano; St. Peter's Hospital at Jacobu, Amansie Central. During this period, patients with suspected MU lesions were identified in the four districts by specially trained personnel, and referred to the district hospitals where they were screened further. A clinical diagnosis was made by the doctor and the patients were then started on rifampicin-streptomycin combination in accordance with the World Health Organisation recommendations.

At Tepa District Hospital confirmation of the diagnosis was done by a physician specialist and his team from Komfo Anokye Teaching Hospital, who reviewed the treatment and took swabs and punch biopsies for polymerase chain reaction (PCR), direct smear examination, culture and histopathology. At the other three hospitals tissue excised at surgery and wound swabs were sent for the above four investigations. Specimen were analysed at either Komfo Anokye Teaching Hospital in Kumasi or the Kumasi Cen- tre for Collaborative Research (KCCR) into Tropical Medicine.The surgical team comprised one plastic surgeon and one theatre nurse from Komfo Anokye Teaching Hospital, in Kumasi, and was assisted by a doctor, anaesthetist, and theatre nurses from the district hospitals. St. Peter's Hospital at Jacobu was not adequately equipped for surgery. Patients in Amansie Central District were operated upon at Bekwai District Hospital in Amansie East, about $35 \mathrm{~km}$ from Jacobu. In each district, patients with ulcers and other lesions diagnosed as MU disease and who had completed eight weeks, or a minimum of four weeks treatment with rifampicinstreptomycin combination, and who had residual lesions were selected for surgical treatment.

The name, age, sex, and location of lesion were recorded; the patients were examined clinically as to their fitness for anaesthesia; the lesion was measured (widest diameter) and a clinical photograph taken. Surgery for lower limb lesions in adults was done under spinal anaesthesia; all other lesions were operated upon under general anaesthesia. Surgery involved: shaving wounds of excess granulation tissue and partial thickness skin grafting, wound excision and immediate skin grafting, wound excision without grafting, wound debridement, sequestrectomy, release of contracture and flap repair. Excised wounds which could not be grafted immediately were grafted after two weeks. No wound or lesion had to be excised more than once.

Skin grafted wounds were inspected after one week during which time the dressing was changed. The wound at the donor site was inspected after two weeks, when the dressing was also changed; thereafter the wounds - donor and recipient sites - were dressed twice a week till all wounds healed. Vaseline impregnated gauze was the dressing material used for both donor and recipient sites. Patients were included in the study if the clinical diagnosis of MU disease has been confirmed by any of: direct smear examination, culture of MU, polymerase chain reaction (PCR) or histopathology; have completed eight weeks or a minimum of four weeks of treatment with rifampicin-streptomycin combination; have undergone surgery for MU disease.

\section{RESULTS}

The study period extended from September 2005 to September 2009 and included 132 patients with MU disease, made up of 51 patients from Tepa District Hospital, 36 from St Martin's Hospital at Agroyesum, 16 from Bekwai District Hospital and 29 from Goaso Government Hospital. There were 67 males and 65 females; the male to female ratio is 1.03: 1 .Their ages ranged from four years to 98 years with a mean age of 29.9 years, with a standard deviation of 20.7 . 
The highest prevalence of MU disease occurred in the age group $11-20$ years; most of the older patients, 14 (10.6\%), aged 50 years and above, were seen at St. Martin's Hospital at Agroyesum, the oldest being 98 years. These were mostly problem cases referred from other endemic districts due to graft failure, recurrent ulcers, and contractures.

Table 1 Age and Sex distribution of MU Patients in 4 Endemic Districts

\begin{tabular}{|l|l|}
\hline Age Group & Total $(\%)$ \\
\hline $0-10$ years & $19(14.4)$ \\
\hline $11-20$ years & $38(28.8)$ \\
\hline $21-30$ years & $23(17.4)$ \\
\hline $31-40$ years & $16(12.1)$ \\
\hline $41-50$ years & $12(9.1)$ \\
\hline $51-60$ years & $12(9.1)$ \\
\hline$>61$ & $12(9.1)$ \\
\hline Total & $132(100)$ \\
\hline Sex & \\
\hline Male & $67(50.8)$ \\
\hline Female & $65(49.2)$ \\
\hline
\end{tabular}

A total of $135 \mathrm{MU}$ lesions were found on the 132 patients. Table 2 shows the distribution of the lesions.

The clinical forms of the disease studied are shown in Table 3. The sizes of the MU lesions seen at the time of diagnosis, before the commencement of rifampicin and streptomycin treatment, were: category I, 10 $(7.41 \%)$; category II, $56(41.48 \%)$; category III, 69 $(59.10 \%)$. At the time of surgery, when the patients had completed the rifampicin and streptomycin treatment, the sizes were: category I, 22 (16.30\%); category II, $46(34.07 \%)$ and category III, 67 $(49.63 \%)$.

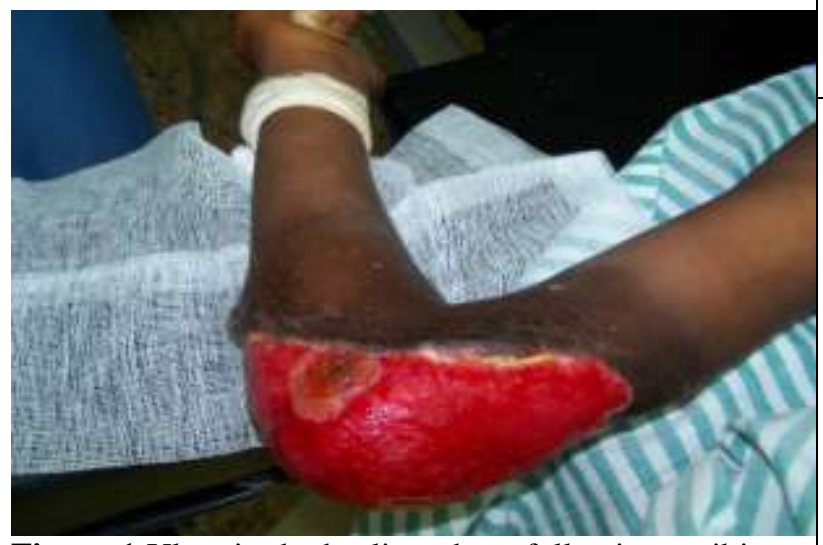

Figure 1 Ulcer in the healing phase following antibiotic treatment
About $92 \%$ of the lesions were ulcers, most of them in the healing phase, after antibiotic treatment; $36(26.0 \%)$ required only skin grafting; $54(39.0 \%)$ required excision and immediate skin grafting. Healing of these lesions occurred by the third week, except for 10 (7.2\%) ulcers, (three from Tepa, seven from Agroyesum) where the grafts failed. The wounds were debrided, and re-grafted after two weeks. The wounds healed by the fourth postoperative week. For 25 ulcers in category I and II excision was the only procedure required; satisfactory healing occurred in four weeks.

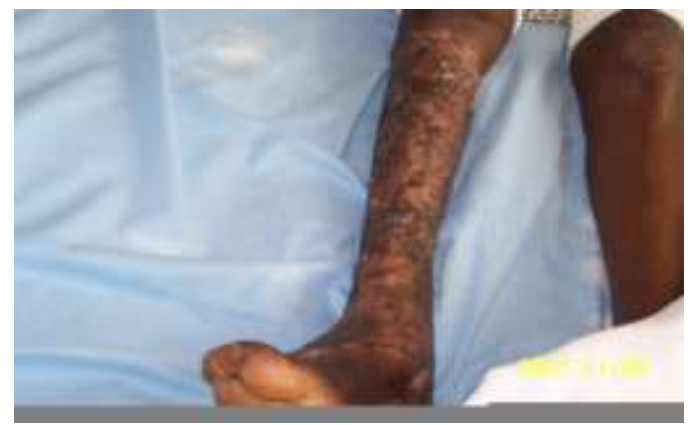

Figure 2 Satisfactory healing of ulcer following skin grafting

Table 2 Sites of Involvement of MU lesions in patients Studied N=135

\begin{tabular}{|l|l|}
\hline SITE & TOTAL (\%) \\
\hline TRUNK & \\
Anterior & $\mathbf{2 ( 1 . 5 )}$ \\
\hline HEAD \& NECK & \\
Face & $\mathbf{1}(\mathbf{0 . 7 )}$ \\
\hline UPPER LIMB & $\mathbf{4 0}(\mathbf{2 9 . 6 )}$ \\
Elbow & 12 \\
Finger & 1 \\
Hand & 9 \\
Upper Arm & 9 \\
Forearm & 7 \\
Wrist & 1 \\
Shoulder & 1 \\
\hline LOWER LIMB & $\mathbf{9 2}(\mathbf{6 8 . 2 )}$ \\
Foot & \\
$\quad$ Sole & 13 \\
$\quad$ Dorsum & 9 \\
Leg & 34 \\
Ankle & 16 \\
Thigh & 9 \\
Popliteal & 2 \\
Region & 1 \\
Calf & \\
Gluteal & 3 \\
Region & \\
Knee &
\end{tabular}


After eight weeks of treatment with antibiotics 10 ulcers, five from Tepa, three from Agroyesum, two from Goaso, were not healing; they were still covered with slough, with pale granulation tissue in the floor. Wound debridement was done; the wounds were dressed with vaseline-impregnated gauze for three weeks before skin grafting could be done. Healing was also gradual and took about four weeks.

Table 3 Clinical forms of Mycobacterium ulcerans Disease $\mathrm{N}=135$

\begin{tabular}{|l|c|c|}
\hline \multicolumn{1}{|c|}{ LESION } & $\mathbf{n}$ & \% \\
\hline Papule & 1 & $0.74 \%$ \\
\hline Nodule & 2 & $1.48 \%$ \\
\hline Oedematous lesion & 4 & $2.69 \%$ \\
\hline Osteomyelitis & 2 & $1.48 \%$ \\
\hline Ulcer & 124 & $91.85 \%$ \\
\hline Contracture & 2 & $1.48 \%$ \\
\hline
\end{tabular}

Bone involvement occurred in two cases in this series: one patient from Tepa who had MU infection of the right hand required sequestrectomy for 2 necrotic metacarpals; one patient from Goaso with a necrotic distal third of the right fibula also had sequestrectomy. Both wounds healed uneventfully (Table 4).

Table 4 Surgical procedures performed for MU patients $\mathrm{N}=139$

\begin{tabular}{|l|l|}
\hline PROCEDURE & TOTAL \\
\hline Excision & $25(18.0 \%)$ \\
\hline Skin grafting & $36(26.0 \%)$ \\
\hline $\begin{array}{l}\text { Excision \& skin } \\
\text { grafting }\end{array}$ & $54(39.0 \%)$ \\
\hline Debridement & $10(7.2 \%)$ \\
\hline Sequestrectomy & $2(1.4 \%)$ \\
\hline Re-grafting & $10(7.2 \%)$ \\
\hline $\begin{array}{l}\text { Contracture } \\
\text { release }\end{array}$ & $2(1.4 \%)$ \\
\hline
\end{tabular}

\section{DISCUSSION}

The management of MU disease has undergone several changes as the pathogenesis of the disease was gradually elucidated.

The distribution of the lesions is consistent with the findings of other workers ${ }^{5,6,9}$ emphasising the prevalence of MU lesions on the limbs, especially the lower limb.

Initially surgical excision was the standard treatment; when performed at the nodular stage it was curative. In most endemic areas patients present late with ulcerated lesions; for such cases wide surgical excision was the treatment of choice. ${ }^{2,4,11,12}$ Adjunctive skin grafting was usually required. ${ }^{4,12,13}$

A clinical trial conducted in Ghana under the auspices of the World Health Organisation demonstrated that after daily treatment with rifampicin and streptomycin for at least four weeks MU could no longer be cultured from the lesions. ${ }^{14}$ The antibiotic treatment reduced the surface area of most lesions by more than $50 \%$, allowing less extensive surgical excision. The $6^{\text {th }}$ World Health Organisation Advisory Committee on MU disease recommended in March 2003 the daily administration of rifampicin and an aminoglycoside usually streptomycin, for eight weeks as the first line of treatment for all forms of Mycobacterium ulcerans disease. ${ }^{7}$

Current recommendations for treatment of MU disease are as follows:

1. A combination of rifampicin and streptomycin/amikacin for eight weeks as a first-line treatment for all forms of the active disease. Nodules or uncomplicated cases can be treated without hospitalisation.

2. Surgery to remove necrotic tissue, cover skin defects and correct deformities.

3. Interventions to minimise or prevent disabilities.

The papules and nodules were excised at St. Martin's Hospital at Agroyesum. This hospital and its district being amongst the first to start active management of Mycobacterium ulcerans disease in Ghana have experienced health workers who are able to detect the disease at an earlier stage. The rarity of the earlier forms of the disease, and especially of the oedematous forms may be due to the efficacy of prior streptomycin-rifampicin treatment, lesions which heal with antibiotics alone are not seen by the surgical team; it may also be due to failure of patients to report for treatment since such lesions are painless and not deforming.

In the present series prior antibiotic treatment did reduce the number of active early disease, leaving only ulcers in the healing phase (Figure 1). Out of a total of 132 patients only four oedematous lesions, two nodules, and one papule were found. The extent of surgery was also reduced; the main clinical form, the ulcers, required mostly skin grafting with very satisfactory healing (fig.2). Though two cases required sequestrectomy, no extensive surgery such as limb amputations were required during the study period. No ulcer needed to be excised more than once as in other series. ${ }^{15}$ In a three year review of management of MU disease at St Martin's Hospital at Agroyesum, Asiedu and Etuaful ${ }^{16}$ saw a total of 102 patients; 36 in 1994, 34 in 1995, and 32 in 1996. 
Surgery alone, without antibiotics, was the accepted mode of treatment: this involved $10(9.8 \%)$ amputations, $65(63.7 \%)$ wound excisions and skin grafting, $25(24.5 \%)$ wound excisions without skin grafting and $2(1.9 \%)$ sequestrectomies. In the current study, there were no amputations; $39.0 \%$ wound excision and grafting; $18 \%$ wound excision and $1.4 \%$ sequestrectomies; simple skin grafting without prior excision could not be done for any of the lesions whereas this constituted $26 \%$ in the current study. This limitation in the extent of surgery seen in the current study, compared to the former, can be attributed to prior antibiotic therapy.

Healing of MU infection occurs by secondary intention after sloughing of epidermis and subcutaneous fat, with later re-epithelialisation. Considerable deformity results from this mode of healing. On the extremities scarring leads to contractures, subluxation of joints, disuse atrophy and distal lymphoedema. ${ }^{2}$ Wound excision and grafting, if performed early in the course of the disease, can prevent or at least minimise the development of contractures. A category three ulcer may heal by antibiotic treatment alone; however since healing is by secondary intention, wound contraction would be excessive, and a contracture may result. ${ }^{17}$ (Figure3) Therefore the suggestion by some researchers ${ }^{9}$ that MU disease could be cured with antibiotics alone should be viewed cautiously in terms of function of the extremity after the lesions have healed. Currently it is known that only early (less than six months old) and smaller (less than $10 \mathrm{~cm}$ diameter) lesions can be cured by antibiotics alone without surgery. ${ }^{18}$

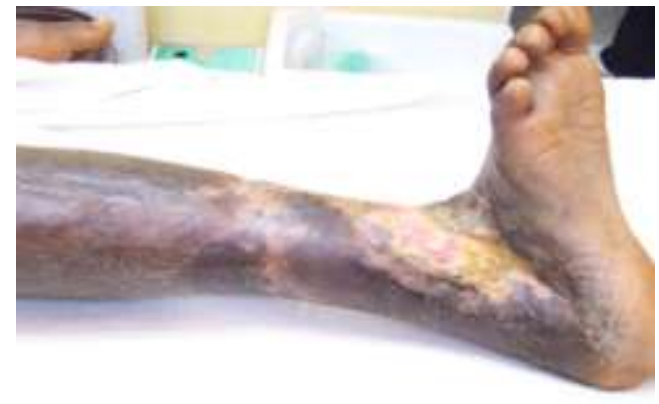

Figure 3 Contracture following healing of ulcer

Finally interventions to minimise or prevent disability should not only emphasise physiotherapy; early medical and surgical intervention can also prevent or minimise the development of contractures, the main cause of disability in Mycobacterium ulcerans patients.

\section{CONCLUSION}

The introduction of antibiotic treatment for Mycobacterium ulcerans disease has improved the management of the condition, and minimised the extent of surgery. The combination of antibiotic treatment and surgical intervention is necessary not only to accelerate healing but also to prevent the development of contractures during healing.

\section{ACKNOWLEDGEMENTS}

The surgical outreach services to Asunafo, AmansieWest, Ahafo-Ano and Amansie Central District were funded exclusively by the National Buruli Ulcer Control Programme.

The surgical team is very grateful to Dr. Richard Philips and his medical team from Komfo Anokye Teaching Hospital; Dr. Akuamoah Boateng and his team from Tepa District Hospital; Dr. O.K. Afreh and his team from Goaso Government Hospital; Dr. M. Forson and his team from Bekwai District Hospital; and the doctors and staff of St. Martin's Hospital at Agroyesum.

\section{REFERENCES}

1. Mork F. Cutaneous Ulcers, Sinuses and Fistulae in: Badoe E.A., Archampong E.Q., da RochaAfodu J.T. (Eds) Principles and Practice of Surgery including Pathology in the Tropics 3rd Eds Ghana Publ. Corp., Accra, 2000: 65 - 76.

2. Horsburgh C.R., Meyers W.M. Buruli Ulcer in: Horsburgh C.R., Nelson A.M. eds: Pathology of Emerging Infections 1997 Am. Soc. Micro. Washington DC 2005 - 4171 Ch 7:119 - 134.

3. WHO Weekly Epidemiological Record no. 17, 2008; 83; 145 - 156.

4. Buntine J. Crofts K. Management of Mycobacterium Ulcerans Disease: WHO Manual for Health Care Providers. World Health Organisation, 2001.

5. Amofah G, Bonsu F, Tetteh C et al Buruli Ulcer in Ghana: Results of a National Case Search. Emerg. Infec. Dis. 2002; 8 (2): 167-170

6. Marston B.J. Diallo M.O. Horsburgh C.R. et al Emergence of Buruli Ulcer Disease in the Daloa Region of Cote d'Ivoire Am. J. Trop. Med. Hyg. 1995; 52: 219 - 224.

7. WHO, 2004 Provisional Guidelines on the Role of Specific Antibiotics in the Management of Mycobacterium Ulcerans Disease (Buruli Ulcer) World Health Organisation, Geneva, Switzerland WHO/CDS/CPE/GBUI/2004 - 10.

8. Sizaire V., Nackers F., Comte E et al Mycobacterium Ulcerans Infection, Control, diagnosis and treatment. Lancet Infect. Dis 2006; 6: 288 296. 
9. Charity A. Ardant M., Adeye A et al Promising Clinical Efficacy of StreptomycinRifampicin combination for Treatment of Buruli Ulcer (Mycobacterium ulcerans) Disease. Antimicrob. Agents Chemother. 2009; 5(11): 4029 - 4035.

10. WHO Fact Sheet No. 199. Buruli Ulcer Disease (Mycobacterium ulcerans Infection) Revised March 2007.

11. Clancey J.K., Dodge O.G., Lunn H.F. et al Mycobacterial Skin Ulcer in Uganda. Lancet 1961; 2: 951 - 954.

12. Glym P.J. The use of Surgery and Local Temperature Elevation in Mycobacterium ulcerans Infection Aust. N. Z.J. Surg. 1972; 41: $312-317$.

13. Muelder K. Nouron A., Buruli Ulcer in Benin Lancet 1990; 336: 1109 - 1111.

14. Etuaful S.B., Carnonnelle J., Grosset S. et al Efficacy of the Combination Rifampicinstreptomycin in Preventing Growth of $M y$ - cobacterium ulcerans in early lesions of Buruli Ulcer in Humans. Antimicrob. Agents Chemother. 2005; 49: 3182 - 3186.

15. Agbenorku P., Agbenorku M. Gota E., et al. The Benefits of a Combination of Surgery and Chemotherapy in the Management of Buruli U1cer Patients J. Sci. Tech. 2006;(26 2) : 6-21.

16. Asiedu K, Etuaful S. Socioeconomic Implications of Buruli Ulcer in Ghana. A Three-year Review. Am J. Trop. Med. Hyg. 1998; 59 (6): 1015-1022

17. Watson A.C.H. Complications of Burns in: Morris A. McG., Stevenson J.H. Watson A.C.H. Eds. Complications of Plastic Surgery. Bailliere Tindall; London 1989; 221 - 241.

18. Nienhuis W.A, Stienstra Y, Thompson W.A, Awuah P.C et al Antimicrobial treatment for early limited Mycobacterium ulcerans infection: a randomised control trial. The Lancet 2010;375 (9715); $664-672$ 